\title{
Structural Response and Reliability Analysis of RC Beam Subjected to Explosive Loading
}

\author{
MAURIZIO ACITO ${ }^{1 a}$, FLAVIO STOCHINO $^{2 b}$, SERGIO TATTONI ${ }^{2 c}$ \\ ${ }^{1}$ Dipartimento di Ingegneria Strutturale, Politecnico di Milano \\ ${ }^{2}$ Dipartimento di Ingegneria Strutturale,Infrastrutturale e Geomatica Università di Cagliari \\ amaurizio.acito@polimi.it, bfstochino@unica.it, ${ }^{\mathrm{c}}$ stattoni@unica.it
}

\begin{abstract}
Keywords: Blast Loading, Monte Carlo Simulation, R.C. beams, Structural reliability.
\end{abstract}
\begin{abstract}
The random nature of the explosion load, associated with the random nature of material properties, and geometric dimensional characteristics, implies the need to consider them into the reliability analysis in order to have a more correct estimation of the structural behavior. Therefore, when the randomness of these parameters in the analysis is considered, the response of the structure assumes probabilistic nature, and this makes it necessary to look into the reliability measure.

This paper presents results from a parametric investigation of the reliability of reinforced concrete (RC) beam subjected to blast load. The probabilistic responses of the maximum displacement for a reinforced concrete flexural member under blast loadings are evaluated by means of nonlinear dynamic analysis with simplified equivalent single-degree-of-freedom (SDOF) system.

Results of numerical simulations have shown the response of structures, in terms of maximum displacement in relation also to the blast load and the geometrical and mechanical characteristics of the beams. Monte Carlo simulation of dynamic response of the equivalent SDOF system is performed to estimate the reliability.
\end{abstract}

\section{Introduction}

In recent years the issue of the structure safety under blast loading has become a dramatic problem. The evaluation of building response is a very complex matter for the strong non-linearity of the involved phenomena.

In the scientific literature there are a lot of examples of nonlinear SDOF response under impulsive loads. For example [1] represents an early work that analyses the nonlinear SDOF response under blast loads for R.C. elements.

Other authors, for example [2], have used nonlinear response hypothesis for a SDOF model. They connected the solutions of differential equation of motion pre-yielding and post-yielding similar to what calculated in [1]. In an earlier work [3], there are statistical comparison between nonlinear dynamical SDOF analysis and FE model analysis referred to R.C. beams under blast load. This comparison shows a good agreement on the results of the two models, characterized by a non-linear elasto - plastic response.

Some authors, for example [4], [1] and [5], claim that in the definition of the models it should be considered the role played by the stirrups in the confinement of compressed concrete, and they use constitutive models that take account of this effect.

In [4] the author had already addressed the issue of structural safety of R.C. building under blast loads in a general way, by recalling the relevant general framework, the fundamental aspects of the characterization of the loading actions and of the response of R.C. beams.

In this paper the authors develop a statistical analysis of R.C. beams safety with a nonlinear SDOF model, in order to highlight significance in the response of the various parameters and variables that govern the problem of RC beams subject to explosion loads. 


\section{Blast Loading}

There are a lot of different kinds of explosion and this leads to considerable complexity and uncertainty in assessing the effects on a structure.

The first issue is the characterization of the action: it depends on the kind of explosion (detonation or deflagration) and on the boundary conditions (internal explosion confined and not confined, external explosion). Therefore the action can be very different both in terms of maximum intensity of the overpressure both in terms of time-history. The popular manuals TM5-1300 - 1990 and FEMA report from $n .426$ to 430 supplies the most significant scenarios definitions.

Hereinafter the authors refer to situations such as those represented in Figure 1.

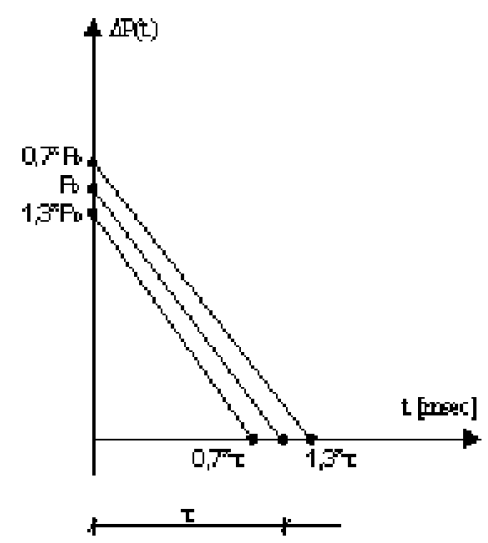

Table 1: Blast scenarios

\begin{tabular}{|c|c|c|c|}
\hline scenarios & High & Medium & Low \\
\hline $\mathrm{P}_{0}[\mathrm{MPa}]$ & 1,6 & 0,16 & 0,016 \\
\hline$\tau[\mathrm{ms}]$ & 2,7 & 27 & 270 \\
\hline
\end{tabular}

Figure 1: Blast scenarios.

From scientific literature arises that in the explosion loads definition there are two main sources of uncertainty. The first is related to the considerable diversity of values of the parameters characterizing the explosion; the second is linked to the significant possibility of plausible scenarios that could be envisaged in terms of position and power (weight of TNT equivalent) of the explosive charge.

The first source of uncertainty can be treated in a classical way assuming the blast parameters as random variables and referring to numerical simulation and full scale testing of literature benchmark problems. The second source of uncertainty can only be managed with risk analysis, which excludes scenarios implausible. In fact, the limited number of the experimental data, the level of danger associated with an explosion, cannot be studied on the basis of statistical data. Moreover, the different scenario conditions determine significant differences between the blast parameters values. In this work the laws stated by Henrych [6] are considered as laws of variation of the overpressure and pulse with scaled distance $Z$.

The numerical simulations are developed from these three scenarios by Monte Carlo variations of the peak pressure $P_{0}$ and the positive phase duration $\tau$ as indicated in Figure 1 .

\section{Structural model}

Constitutive law of concrete and steel. In order to evaluate the response of R.C. bending elements it is important to estimate the increasing of deformability offered to the concrete by an adequate transversal reinforcement. In the present work it was considered significant to refer to the EC2 model. Even for the steel the authors use the constitutive models of EC2 as shown in Figure 2.

The strength of concrete considered in this work by the authors varies between $\mathrm{f}_{\mathrm{c}}=25 \mathrm{MPa}$ and $\mathrm{f}_{\mathrm{c}}=35 \mathrm{MPa}$. Reinforcement steel is supposed to have yield strength varying between $\mathrm{f}_{\mathrm{y}}=420 \mathrm{MPa}$ and $\mathrm{f}_{\mathrm{y}}=480 \mathrm{MPa}$. 

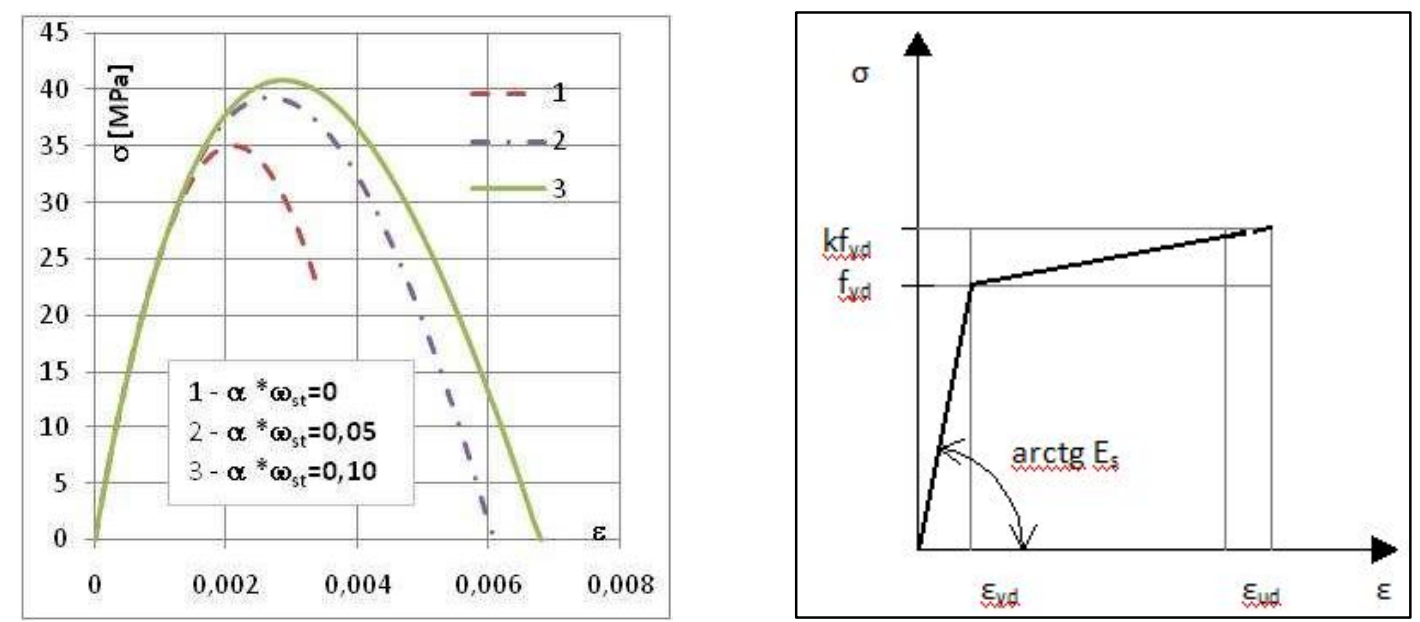

Figure 2: EC2 constitutive law for concrete in relation to the confinement and for steel.

Structural and sectional models in quasi-static condition. In order to define a structural model of a bending element it is necessary to create a sectional bending moment-curvature model. Taking into account that the deformation caused by a blast load is very significant, it is suitable to approximate the $\mathrm{M}-\theta$ diagram with a simply two sided line that covers stages I and II. In [1] it was considered that, after the crushing of the concrete cover, it enters in stage III and it may be expelled. Consequently the effective height $d$ is reduced.

From sectional behavior it is obtained the structural one by integration of the curvature along the beam [1] or, in a rough calculation, by the analysis of a rigid-plastic mechanism (Figure 3).
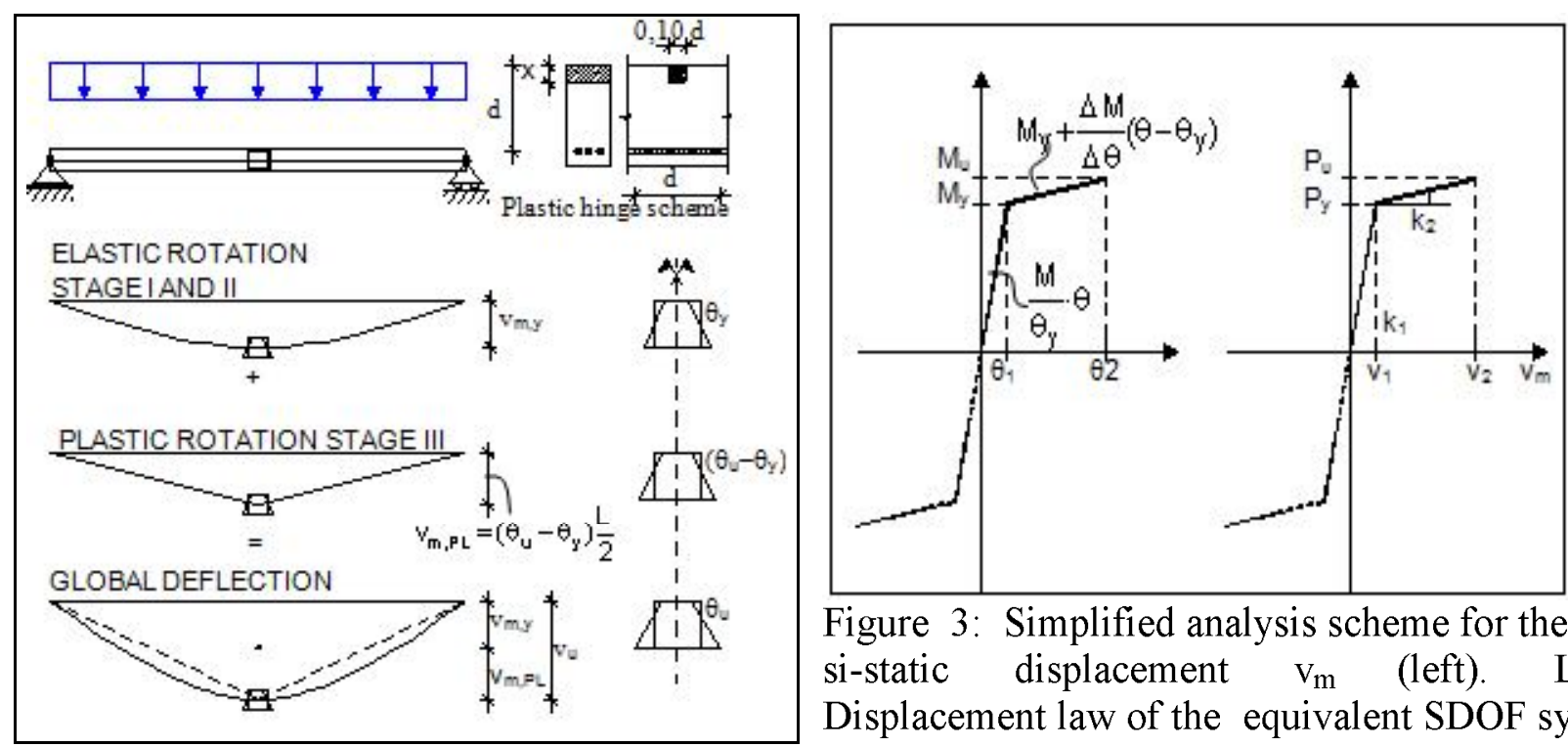

Figure 3: Simplified analysis scheme for the quasi-static displacement $\mathrm{v}_{\mathrm{m}} \quad$ (left). LoadDisplacement law of the equivalent SDOF system (right).

\section{SDOF Model}

In blast or shock analysis, it is established experience that the first peak of displacement is the more severe condition [1], [2]. Subsequent cycles show significant decreases in intensity and the oscillations dampen quickly. Therefore, it is customary to refer to the response in terms of maximum deformation related to the first peak of displacement caused by impulsive load, since it is unlikely that the structure collapses in successive peaks. To do this, a one degree of freedom system (SDOF) is suitable for a simple and reliable analysis, especially for symmetrical elements in bending. Figure 4. shows a SDOF model where $\mathrm{M}_{\mathrm{E}}$ is the equivalent mass, $\mathrm{k}_{\mathrm{E}}$ is the equivalent stiffness and $\mathrm{D}_{\mathrm{E}}$ is the equivalent damper. 


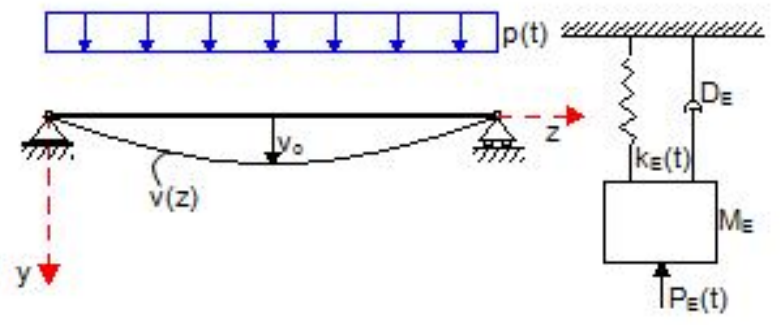

Figure 4: Scheme of SDOF system for a simple supported beam.

Equivalence criteria. The equivalent mass $\mathrm{M}_{\mathrm{E}}$ of the SDOF is quantified from the structural characteristics through energy considerations. Referring to a simple supported beam (Figure 4)in [1] the author assumes $\mathrm{M}_{\mathrm{E}}=0.769 \mathrm{M}_{\mathrm{TOT}}$, where $\mathrm{M}_{\mathrm{TOT}}$ is the total mass of the beam and any weight applied. Again with reference to the diagram of Figure 4, with similar energy considerations, the equivalent load on the SDOF is determined by:

$$
P_{E}=\frac{P}{V_{0}} \int_{0}^{L} v(z) d z=\frac{2}{3} p \cdot L
$$

The equivalent spring represents the bending stiffness of the beam, through the relation loaddisplacement as the one previously represented. It expresses the nonlinear elastic force developed as the displacement of the mass $\mathrm{M}_{\mathrm{E}}$ increases. With reference to previous figures it is possible to indicate the values of equivalent stiffness that is the slope of the two branches of the forcedisplacement law, where $\beta$ is a coefficient that depends on the boundary conditions.

$$
k_{1}=k_{y}=\frac{P_{y}}{v_{1}}=\beta \cdot \frac{E_{I} J_{I I}}{L^{3}} \quad k_{2}=\frac{P_{u}-P_{y}}{v_{2}-v_{1}}
$$

The equivalent dumper as been neglected because in this analysis only the first peak of oscillation was considered, so $\mathrm{D}_{\mathrm{E}}=0$.

Solution of the nonlinear dynamic problem. To solve the dynamic problem the authors referred to the approach proposed in [1] and [7]; the equations of motion has been solved taking into account the elastic and elasto-plastic field with appropriate initial conditions.

Table 2: Geometrical and strength characteristics.

\begin{tabular}{|c|c|c|c|c|c|c|c|c|}
\hline$(1)$ & $(2)$ & $(3)$ & $(4)$ & $(5)$ & $(6)$ & $(7)$ & $(8)$ & $(9)$ \\
\hline $\mathrm{h} / \mathrm{L}$ & $\begin{array}{c}\mathrm{L}[\mathrm{m}] \\
\mathrm{ll}=100 \mathrm{~cm}\end{array}$ & $\mathrm{~b}$ & $\rho_{\mathrm{s} 1}=\frac{\mathrm{A}_{\mathrm{s} 1}}{\mathrm{~b} \cdot \mathrm{d}}$ & $\mathrm{u}=\frac{\mathrm{A}_{\mathrm{s} 2}}{\mathrm{~A}_{\mathrm{s} 1}}$ & Stirrups & $\mathrm{p}_{\mathrm{st}}[\mathrm{cm}]$ & Concrete & Steel \\
\hline $1 / 9$ & $6 \div 12$ & $\mathrm{~h} / 2.5$ & $0.005 \div 0.01$ & $0.25 \div 0.5$ & $\phi 8$ & $20 \div 30$ & $\mathrm{fc}=25 \div 35$ & $\mathrm{fy}=420 \div 480$ \\
\hline $1 / 10$ & $6 \div 12$ & $\mathrm{~h} / 2.5$ & $0.005 \div 0.01$ & $0.25 \div 0.5$ & $\phi 8$ & $20 \div 30$ & $\mathrm{fc}=25 \div 35$ & $\mathrm{fy}=420 \div 480$ \\
\hline $1 / 11$ & $6 \div 12$ & $\mathrm{~h} / 2.5$ & $0.005 \div 0.01$ & $0.25 \div 0.5$ & $\phi 8$ & $20 \div 30$ & $\mathrm{fc}=25 \div 35$ & $\mathrm{fy}=420 \div 480$ \\
\hline $1 / 12$ & $6 \div 12$ & $\mathrm{~h} / 2.5$ & $0.005 \div 0.01$ & $0.25 \div 0.5$ & $\phi 8$ & $20 \div 30$ & $\mathrm{fc}=25 \div 35$ & $\mathrm{fy}=420 \div 480$ \\
\hline $1 / 13$ & $6 \div 12$ & $\mathrm{~h} / 2.5$ & $0.005 \div 0.01$ & $0.25 \div 0.5$ & $\phi 8$ & $20 \div 30$ & $\mathrm{fc}=25 \div 35$ & $\mathrm{fy}=420 \div 480$ \\
\hline $1 / 14$ & $6 \div 12$ & $\mathrm{~h} / 2.5$ & $0.005 \div 0.01$ & $0.25 \div 0.5$ & $\phi 8$ & $20 \div 30$ & $\mathrm{fc}=25 \div 35$ & $\mathrm{fy}=420 \div 480$ \\
\hline $1 / 15$ & $6 \div 12$ & $\mathrm{~h} / 2.5$ & $0.005 \div 0.01$ & $0.25 \div 0.5$ & $\phi 8$ & $20 \div 30$ & $\mathrm{fc}=25 \div 35$ & $\mathrm{fy}=420 \div 480$ \\
\hline
\end{tabular}

Randomized Numerical Values. The geometrical characteristics and their range of variation are reported in Table 2 . The range has been chosen among the most frequent values in common buildings. These values vary with uniform random distribution model. Stirrups satisfy the minimum requirement of standards (EC 2). In the mass of the beams a permanent load is included equivalent to $40 \mathrm{kN} / \mathrm{m}$. 
For each span length and slenderness geometrical and strength data reported in Table 2 are made varied randomly following the Monte Carlo method within the range indicated. So there were performed $7 \times 7 \times 3000=147000$ runs of the SDOF model for each load scenario (high, medium, low as defined in Table 1).

\section{Results and Conclusions}

Often, in the specialized manuals, the ultimate displacement is defined as a ratio of the span (i.e. $\mathrm{V}_{2}=\mathrm{L} / 50$, or generally $\mathrm{L} / \mathrm{n}$ ) independently of other characteristics of the beam. For R.C. beams the deflection limit as been defined by $v_{1}$ and $v_{2}$ (respectively elastic and ultimate). In Figure 5 , as it could be expected, it is shown that the ultimate limit $\left(\mathrm{v}_{2}\right)$ is a linear function of both span and slenderness: the factor " $n$ " increases with increasing $L$ and decreasing slenderness $L / h=L / d$.
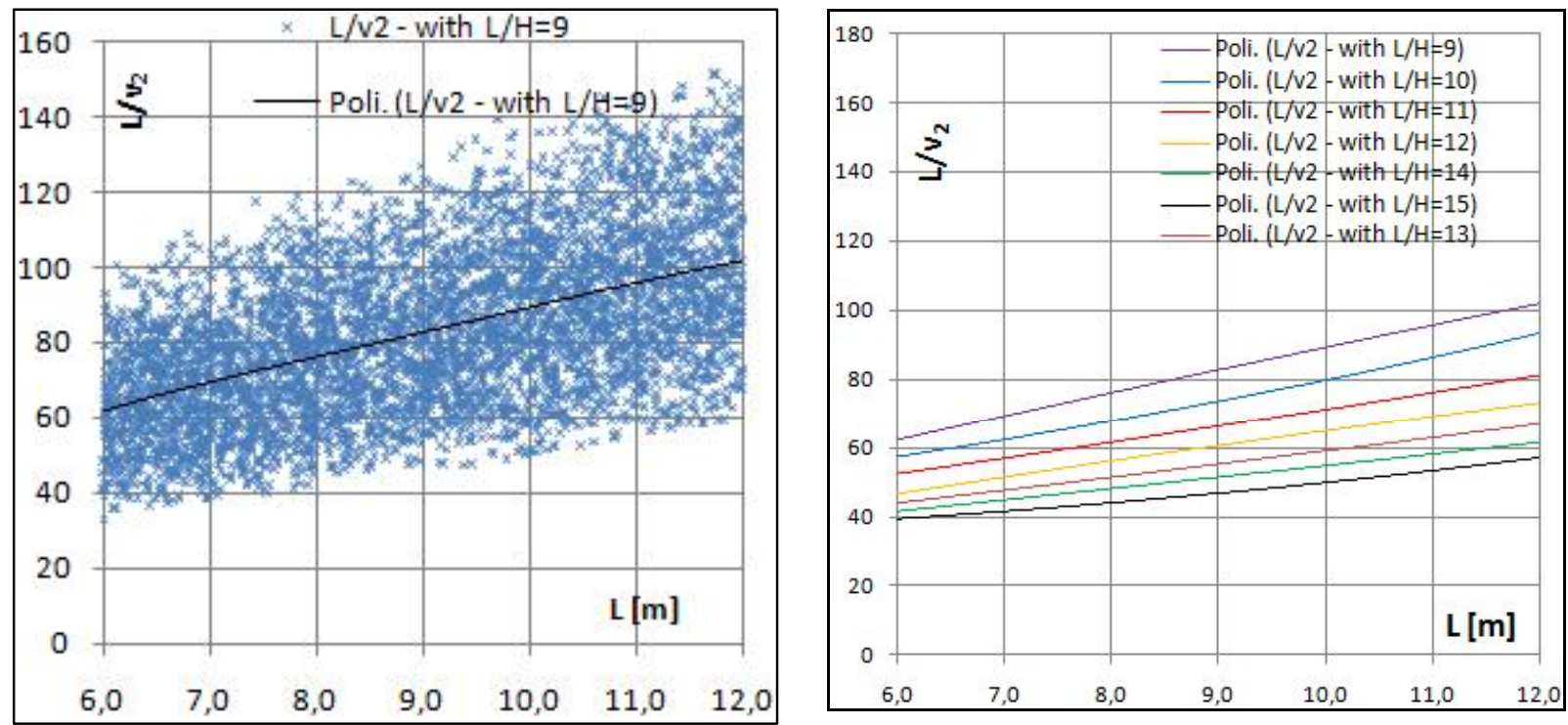

Figure 5: Trend of the ultimate displacement as a function of span and slenderness.
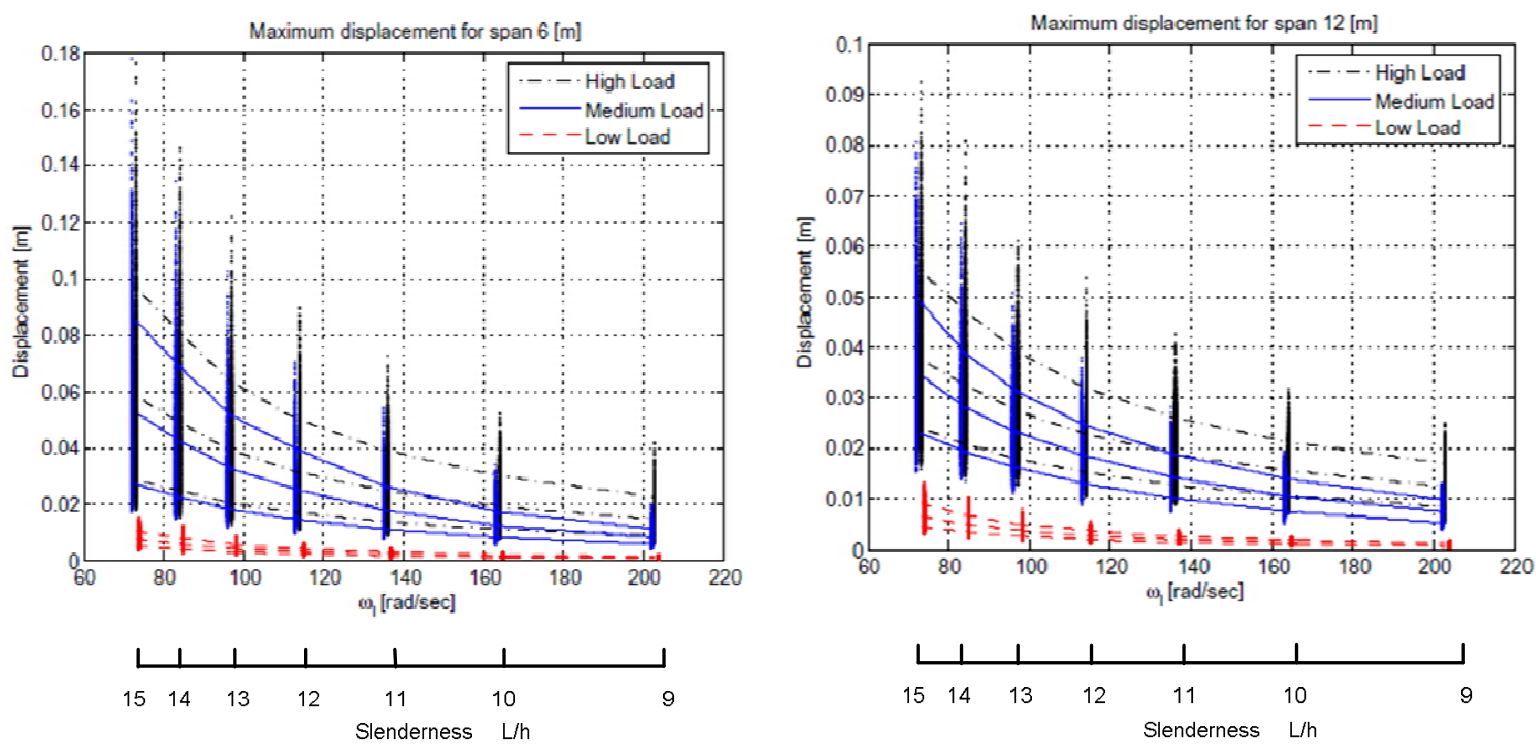

Figure 6: Maximum displacement for 6 and $12 \mathrm{~m}$ span beams. For each load conditions the medium value and the $10 \%$ and the $90 \%$ fractiles are represented.

Figure 6 shows the Maximum displacement results for the shortest and the longest beams that have been analyzed. First of all, the trend as a function of slenderness $\mathrm{L} / \mathrm{h}$ it's similar for both spans. For other span lengths values are between these extremes. In all cases maximum displacement decreases with increasing span length and with decreasing slenderness. This is due to the fact 
that the stiffness of the structural elements is strictly related to the slenderness (more the slenderness more the deformability) and to the scale factor $\alpha=\mathrm{L} / \mathrm{L}_{0}$ (sectional inertia $\mathrm{J}$ and ultimate resisting moment $\mathrm{M}_{\mathrm{Rd}}$ increase proportionally to $\alpha^{3}$ ).

Independently from the load level all distributions of displacement results show a non-symmetric shape. This fact is easy to note from Figure 6, and in particular by calculations of skewness and kurtosis of all distributions (which respectively varies from 0.42 to 1.51 and from 2.69 to 6.30 ).

By comparing Figure 5 and Figure 6 it is clear that under the considered load conditions the ultimate displacement $v_{2}$ is rarely exceeded. But this limit, even if it is acceptable for the integrity of the structural elements, not necessary assures safety for non-structural elements and people. For this aim a more restrictively limit should be considered as for example $\Delta_{\mathrm{MAX}}=\mathrm{L} / 150$. The reliability of a beam under this condition intended as the percentage of overshoot of the above mentioned limit is represented in Figure 7.
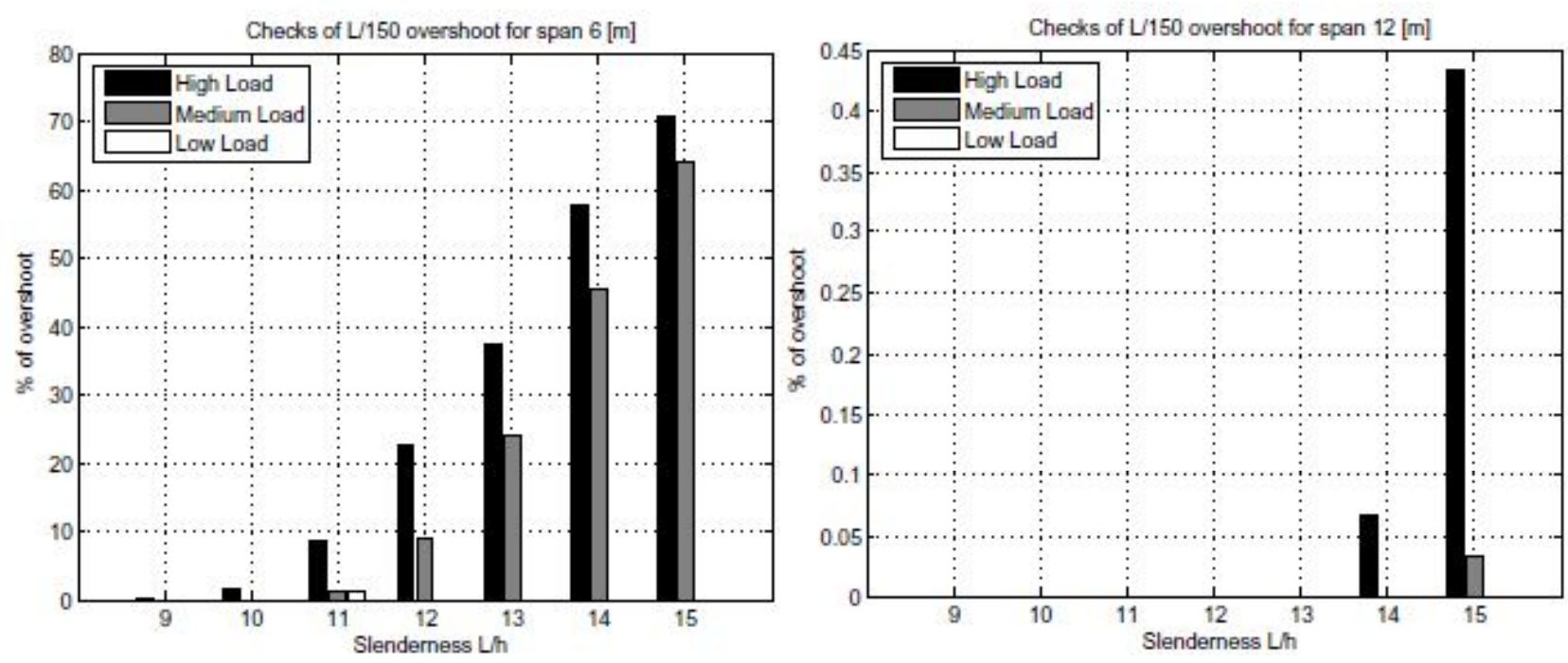

Figure 7: Overshoot of $\mathrm{L} / 150$ limit.

The reliability of a beam depends not only from the slenderness but even from span length. Actually with the same conditions a short span beam is less reliable than a long span one. Having spoken before about the variation of sectional inertia $J$ and resisting moment $\mathrm{M}_{\mathrm{Rd}}$, now it has to be considered the importance of the mass that considerably increases with span length. For these reasons beams with longer span have less probability to exceed the imposed deflection limit.

Further developments of this research are expected with regard to the velocity achieved under blast load and consequent modifications of material strength with dynamic strain-rate.

\section{References}

[1] S. Tattoni, Risposta in regime non lineare di elementi in calcestruzzo ad azioni di tipo impulsivo - Studi e Ricerche - Italcementi SpA - Bergamo Editrice. Vol. 5 - 1983.

[2] Hsin Yu Low, Hong Hao Reliability analysis of reinforced concrete slabs under explosive loading. Structural Safety 23- 2001.

[3] H. C. Rong e B. Li Probabilistic response evaluation for RC flexural members subjected to blat loadings - Structural Safty 29 - 2007.

[4] M.Acito, Sulla sicurezza delle strutture in calcestruzzo armato esposte ad azione da esplosione, Proc. Giornate AICAP 09- Pisa 14-15-16 maggio 2009.

[5] P. Mendis, The 21st Biennial Conference, Concrete in the 3rd Millennium - Concrete Institute of Australia -Brisbane 17-19 Jul 2003

[6] Henrych J. The dynamics of explosion and its use. Amsterdam: Elsevier, 1979.

[7] S. Tattoni, A. Gasperi, Dynamic of non-linear single degree of freedom systems, Proc. of the conference held at University College Swansea 2nd-5th September, 1980. 
Performance, Protection and Strengthening of Structures under Extreme Loading doi:10.4028/www.scientific.net/AMM.82

Structural Response and Reliability Analysis of RC Beam Subjected to Explosive Loading doi:10.4028/www.scientific.net/AMM.82.434 